\title{
AVALIAÇÃO DOS GASES FORMADOS PELO PROCESSO DE PIRÓLISE E PÓS-COMBUSTÃO DE RESÍDUO SÓLIDO URBANO COMO FONTE ALTERNATIVA DE ENERGIA
}

\author{
V. F. DA SILVA ${ }^{1 *}$, L. BATISTELLA ${ }^{1}$, R.C. SUSIN, C. A. ALTHOFF² e H. J. JOSE ${ }^{1}$ \\ ${ }^{1}$ Universidade Federal de Santa Catarina, Departamento de Engenharia Química e Engenharia de \\ Alimentos. \\ ${ }^{2}$ Albrecht Industria Equipamentos Ltd., BR-101, km 29, Pirabeiraba, Joinville, SC, Brasil \\ *E-mail para contato: valdemarfilho21@hotmail.com
}

\begin{abstract}
RESUMO -O trabalho teve como objetivo utilizar o processo de conversão térmica: pirólise acoplado ao processo de pós-combustão, para realizar uma avaliação preliminar dos gases formados com a queima do RSU. A utilização do processo de pirólise teve por finalidade manter uma temperatura ótima, a fim de que não houvesse o desprendimento do cloro no RSU, assim o cloro não seria liberado na pirólise. Já o processo de póscombustão teve o intuito de queimar todos os gases formados provenientes da pirólise. $\mathrm{O}$

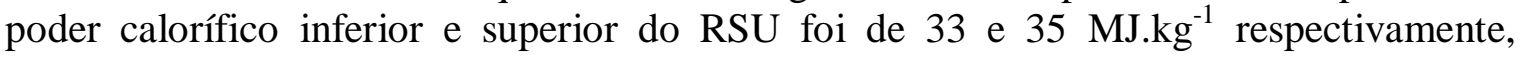
enquanto que a matéria volátil apresentou um alto teor de 82,17\%. O char apresentou teores de $\mathrm{Al}, \mathrm{Fe}, \mathrm{Cl}$ e As de 4,23, 2,18, 4,93 e 0,076 \%, respectivamente. As concentrações dos gases emitidos em mg.N.m ${ }^{-3}$ foram: 36,47 de CO; 18,55 de $\mathrm{SO}_{2}$; 106,86 de $\mathrm{NO}_{\mathrm{x}} ; 3,25 \cdot 10^{-3}$ de BTEX; e 0,039 $\mu \mathrm{g} . \mathrm{N} \cdot \mathrm{m}^{-3}$ de HPA. Em suma, a avaliação dos gases apresentam baixos percentuais comparados com os limites das legislações pertinentes.
\end{abstract}

\section{INTRODUÇÃO}

O desenvolvimento populacional bem com o padrão de vida atual contribuem para o crescimento dos resíduos, como o aumento da utilização de matérias descartáveis. Desta maneira, é preciso ter um plano de desenvolvimento para solucionar o problema em questão, uma vez que não haverá espaço físico (aterros sanitários) para tanto resíduo (COSTA et al. 2012).

Segundo Miskolczi et al. (2011) o resíduo sólido urbano (RSU) é matéria-prima em potencial para a produção de energia renovável. O RSU consiste em diferentes componentes de resíduos, tais como papéis, plásticos, resíduos orgânicos e outros coletados. Alguns dos fatores desfavoráveis do RSU são os seus elevados custos de transporte (baixa densidade), além da presença de cloro em sua composição.

De acordo Mckay (2002) o processo de combustão além de reduzir o volume do resíduo, também pode ser uma alternativa para a geração de energia. A combustão pode ser bem vantajosa, mas a queima do RSU pode resultar em problemas associados à emissão de poluentes, já que o RSU 


\section{9 a 22 de outubro de 2014 \\ Florianópolis/SC}

pode conter em sua composição a presença de cloro originado do PVC. Uma alternativa à combustão de RSU é a pirólise acoplada ao processo de pós-combustão. Segundo Malkow (2004) estes processos são atraentes para reduzir e evitar a formação de corrosão através da retenção do alcalóide, metais pesados, enxofre e cloro dentro os resíduos do processo, evitando a formação de PCDD/F e reduzindo a formação de $\mathrm{NO}_{\mathrm{x}}$ térmico, devido às temperaturas mais baixas e condições redutoras.

Este trabalho teve como objetivo utilizar o processo de conversão térmica: pirólise acoplado ao processo de pós-combustão para realização de uma avaliação preliminar dos gases formados com a queima do RSU. O primeiro processo teve como objetivo manter uma temperatura ótima, a fim de que não houvesse a liberação do cloro no RSU, assim o cloro não seria liberado na pirólise, e sim retido no char formado. Os gases formados na pirólise seriam queimados nas três câmaras de póscombustão, e ao final da pós-combustão, os gases seriam comparados com as legislações pertinentes.

\section{MATERIAIS E MÉTODOS}

O resíduo e o gerador de calor utilizados foram cedidos pela empresa Albrecht Equipamentos Industriais S.A. localizada na cidade de Joinville-SC. O RSU foi composto em sua maioria de plástico, e pequeno percentual de papel e metal. O plástico foi constituído de uma mistura de vários polímeros, entre eles se destacava o policloreto de vinila (PVC), característico por apresentar cloro em sua composição, sendo este um precursor da formação de poluentes ambientais.

\subsection{Preparação e caracterização do material}

O resíduo foi triturado e peneirado com diâmetro de partículas de $210 \mu \mathrm{m}$. Para caracterização foram utilizadas as seguintes normas: elementar (ASTM D5373-02 para CHN; ASTM D4239 para o enxofre), imediata (ASTM E 1131-03 Shimadzu modelo DTG - 60), cloreto (norma: DIN 15289: 2011) e poder calorífico (ASTM D2015).

\subsection{Ensaio da queima no gerador de calor}

O gerador de calor utilizado foi constituído por: um sistema de alimentação, uma câmara primária (onde ocorreu a pirólise), uma câmara secundária (composta por três câmaras de póscombustão), um sistema de refrigeração, um lavador de gases e uma chaminé (local de saída dos gases). Nos ensaios o lavador de gases não foi utilizado. A Figura 1 ilustra o modelo do gerador de calor.

As análises dos gases formados no processo foram realizadas através de três ensaios, a fim de que houvesse confiabilidade nos resultados. Os ensaios foram realizados com queima do RSU mais cavaco de madeira $(\mathrm{CM})$ na mesma proporção. Para os ensaios foram utilizados uma razão ar/combustível experimental de $11,55 \mathrm{~kg}_{\mathrm{ar}} / \mathrm{kg}_{\text {comb }}$ e uma alimentação de 40 a $70 \mathrm{~kg} \cdot \mathrm{h}^{-1}$.

O equipamento consistiu em dois estágios: o processo de pirólise e o processo de póscombustão. O processo de pirólise foi ajustado a uma temperatura ótima, afim de que o cloro presente no RSU não fosse liberado, e sim ficasse retido no produto sólido (char). Desta forma, o cloro retido 
no char evitaria a formação de poluentes, tais como $\mathrm{HCl}$ e dioxinas e furados. E a pós-combustão foi ajustada para a queima dos gases formados no processo de pirólise. Abaixo uma breve descrição Do processo.

Inicialmente, o resíduo passou por um processo de pirólise, em uma câmara primária, que foi composta por grelhas móveis do tipo recíprocas (movimento de frente para trás). A câmara primária trabalhava em temperaturas de aproximadamente $300{ }^{\circ} \mathrm{C}$, e com uma alimentação de oxigênio subestequiométrica, para que algumas substâncias presentes se tornassem gases e outras assumissem a forma de pequenas partículas. Neste processo dois produtos foram formados: o produto sólido (char), que foi enviado a um receptor externo (ponto 8 da Figura 1), e a fase gasosa que foi encaminhada para a câmara secundária. Na câmara secundária (pós-combustão) a mistura de gases foi então queimada a uma temperatura mais alta por um intervalo de tempo suficiente para que houvesse a combustão completa. Desta maneira, a atmosfera foi altamente oxidante e a temperatura variando normalmente entre $900-1000{ }^{\circ} \mathrm{C}$. Os gases saíram das câmaras de pós-combustão e foram resfriados, e em seguida analisados e lançados para atmosfera, através da chaminé (ponto 12 da Figura 1).

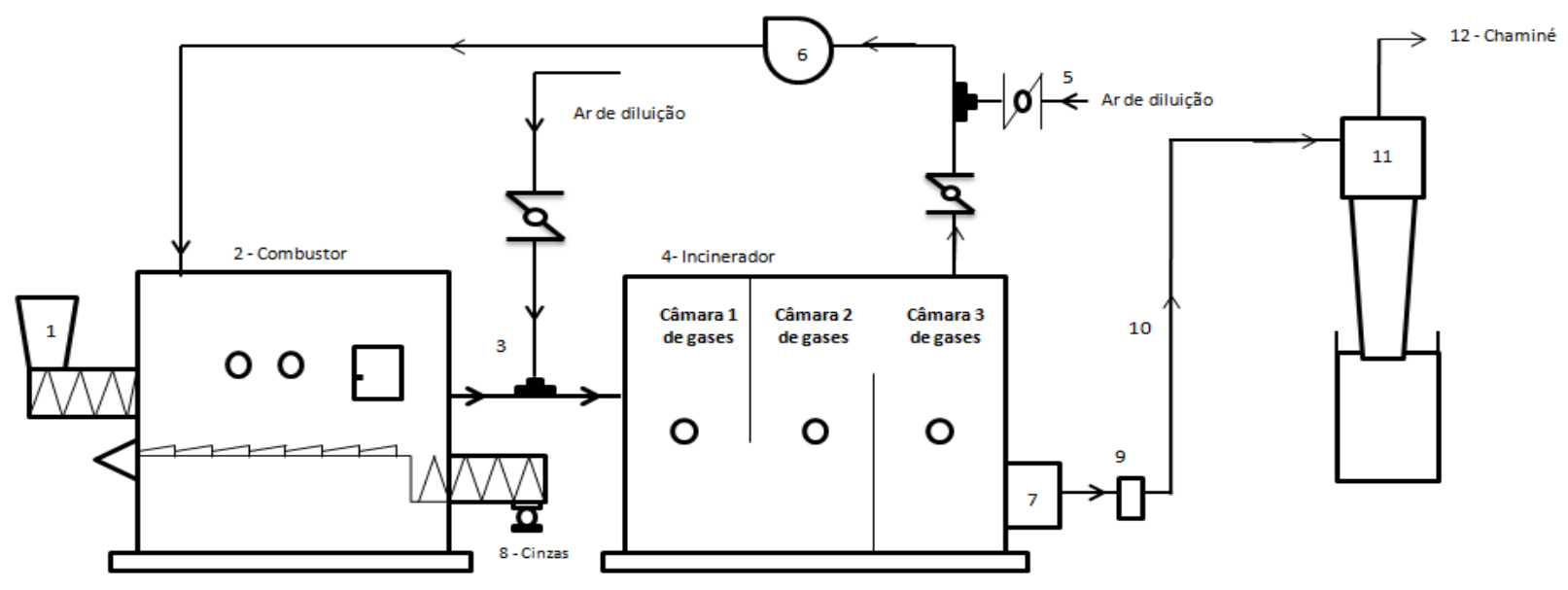

Figura 1: Ilustração da planta piloto: gerador de calor.

1- Alimentação; 2- Câmara primária (pirólise); 3- Exaustão com entrada de ar primário; 4- câmaras de póscombustão; 5-Corrente de ar de recirculação com entrada de ar secundário; 6- Ventilador; 7- Exaustão; 8- Descarga de sólidos; 9- Ponto de amostragem; 10- sistema de refrigeração; 11- Lavador de gases; 12- Saída dos gases (Chaminé).

\subsection{Caracterização dos produtos formados no gerador de calor}

Produtos gasosos: os gases de combustão foram coletados seguindo o método da USEPA 4. As amostras dos gases foram coletadas com um analisador de gases de combustão modelo Greenline MK2, (Eurotron Italiana S.r.1.) a partir da imersão de uma sonda, contendo um termopar acoplado, no ponto de amostragem (ponto 9 na Figura 1). O analisador de gases, foi operado de forma contínua, organizado em um grupo de células eletroquímicas, para análises de $\mathrm{O}_{2}, \mathrm{CO}, \mathrm{NO}_{\mathrm{x}}, \mathrm{SO}_{2}$, e peristor 
para $\mathrm{C}_{\mathrm{x}} \mathrm{H}_{\mathrm{y}}$. $\mathrm{O} \mathrm{CO}_{2}$ foi calculado com bases em combustível previamente definido pelo equipamento. As coletas de BTEX (Benzeno, Tolueno, Etilbenzeno e Xilenos) e HPA foram realizadas segundo recomendações do método USEPA 17 e USEPA 23, respectivamente.

Produto sólido: O resíduo sólido (char) foi preparado e caracterizado.

\section{RESULTADOS E DISCUSSÃO}

\subsection{Caracterização dos resíduos}

Para uma queima eficiente primeiramente foi necessário conhecer as propriedades dos resíduos, a Tabela 1 apresenta os resultados da caracterização de CM, RSU, RSU+CM e do char (RSU+CM).

Tabela 1- Composição dos residuos

\begin{tabular}{|c|c|c|c|c|}
\hline & CM & RSU & RSU+CM & Char $(\mathrm{RSU}+\mathrm{CM})^{3}$ \\
\hline \multicolumn{5}{|c|}{ Análise Elementar } \\
\hline $\mathrm{C}\left[\%\right.$, b.s. $\left.{ }^{1}\right]$ & 49,69 & 69,80 & 55,19 & - \\
\hline $\mathrm{H}[\%$, b.s. $]$ & 5,42 & 10,64 & 6,10 & - \\
\hline $\mathrm{N}[\%$, b.s. $]$ & 0,21 & 0,30 & 0,10 & - \\
\hline S [\%, b.s. $]$ & 0,01 & 0,10 & 0,10 & - \\
\hline $\mathrm{O}^{2}[\%$, b.s. $]$ & 35,30 & 0,76 & 19,68 & - \\
\hline $\mathrm{Cl}[\%$, b.s. $]$ & 0,04 & 5,00 & 5,00 & 4,93 \\
\hline \multicolumn{5}{|c|}{ Análise Imediata } \\
\hline Cinzas $[\%$, b.s.] & 9,33 & 13,40 & 13,83 & 42,50 \\
\hline Matéria volátil [\%, b.s.] & 73,75 & 82,17 & 75,98 & 27,70 \\
\hline Carbono fixo $[\%$, b.s. $]$ & 16,92 & 4,43 & 10,19 & 29,80 \\
\hline Umidade $[\%$, bruta $]$ & 8,45 & 3,99 & 6,59 & 11,26 \\
\hline \multicolumn{5}{|c|}{ Poder Calorífico } \\
\hline PCS [b.s., MJ.kg-1] & 17,70 & 35,44 & 23,03 & - \\
\hline PCI [b.s., MJ.kg ${ }^{-1}$ ] & 16,53 & 33,14 & 21,50 & - \\
\hline
\end{tabular}

${ }^{1}$ :base seca; ${ }^{2}:$ Valor resultante por diferença; ${ }^{3}:$ Resultados dos três ensaios de queima.

Por meio da Tabela 1 foi observado que tanto o RSU bem como o RSU+CM apresentaram alto percentual de carbono e hidrogênio. Mediante aos baixos percentuais de nitrogênio e enxofre, a queima do RSU+CM não formaram altos teores de $\mathrm{NO}_{\mathrm{x}}$ e $\mathrm{SO}_{2}$.

Conforme apresentado na Tabela 1, os resíduos mostraram altos percentuais de matéria volátil. $\mathrm{O}$ alto valor de matéria volátil favorece a maior velocidade de queima e menor estabilidade da chama. Foi observado, que a quantidade de cinza não variou significantemente, com adição do CM ao RSU, porém houve aumento na quantidade de carbono fixo. Por meio da análise do poder calorífico, observou que o RSU (33,14 MJ/kg) apresenta percentual maior que o RSU+CM (21,50 MJ/kg), entretanto o RSU+CM foi o resíduo utilizado no processo de queima devido à logística, uma vez que a adição do $\mathrm{CM}$ ao RSU favorece o aumento na densidade do material, desta forma diminuindo o 
custo com transporte do resíduo ao local de queima.

O percentual de cloro no char foi de $4,93 \%$, desta maneira houve um pequeno percentual de $0,07 \%$ de cloro que foi liberado no processo de pirólise, assim o char ficou rico em cloro, e 0,07\% de cloro foram queimados no processo de pós-combustão.

Segundo as análises imediatas do RSU+CM e do char foram observadas um aumento no percentual de carbono fixo: $10,19 \%$ para $29,80 \%$, enquanto a matéria volátil caiu de $75,98 \%$ para $27,70 \%$, produzindo um resíduo carbonoso (char) rico em carbono.

\subsection{Ensaio de queima no gerador de calor}

As concentrações dos gases $\mathrm{CO}, \mathrm{NO}_{\mathrm{x}}, \mathrm{SO}_{2}$ e BTEX medidas durante os três ensaios de pirólise e pós-combustão dos voláteis, corrigidas a $7 \%$ de $\mathrm{O}_{2}$ de referência para fins de comparação com os limites de emissão, estão apresentadas na Tabela 2.

Tabela 2 - Concentração dos gases corrigidas para $\mathrm{O}_{2 \text { ref }}=7 \%$

\begin{tabular}{ccccc}
\hline [mg.Nm $^{-3}$ ] & CO & NO $_{\mathbf{x}}$ & $\mathbf{S O}_{\mathbf{2}}$ & BTEX \\
\hline RSU+CM & $43,80 \pm 14,40$ & $111,74 \pm 11,61$ & $32,39 \pm 19,70$ & 0,00768 \\
RSU+CM & $36,39 \pm 33,72$ & $110,42 \pm 20,33$ & $21,41 \pm 9,90$ & 0,00207 \\
RSU+CM & $29,21 \pm 14,72$ & $98,42 \pm 13,55$ & $1,85 \pm 2,37$ & - \\
Média & 36,47 & 106,86 & 18,55 & 0,00488 \\
\hline \multicolumn{5}{c}{ Legislaçã 0} \\
\hline CONAMA 316/02 & 125,00 & 560,00 & 280,00 & - \\
17.BlmSchV (24 h) & 70,00 & 280,00 & 70,00 & 14,00 \\
Danish EPA & n.e ${ }^{1}$. & 560,00 & 560,00 & - \\
U. S. EPA & 196,00 & 796,00 & 57,00 & - \\
\hline \multicolumn{5}{c}{ ': Limite não emitido. }
\end{tabular}

Por meio da Tabela 2, foi observado que a concentração de $\mathrm{NO}_{\mathrm{x}}$ foi alta, que pode ser justificado devido a quantidade de ar em excesso presente do processo, uma vez que o resíduo RSU+CM não apresenta alto percentual de nitrogênio, conforme Tabela 1. O percentual de $\mathrm{CO}$ e $\mathrm{SO}_{2}$ emitidos foram baixos: $36,47 \mathrm{mg} \cdot \mathrm{Nm}^{-3}$ de $\mathrm{CO}$ e $18,55 \mathrm{mg} \cdot \mathrm{Nm}^{-3}$ de $\mathrm{SO}_{2}$, respectivamente, devido à combustão completa no processo, e o baixo teor de enxofre $(0,10 \%)$ presente na composição do RSU+CM. As condições de queima fizeram com que as concentrações médias dos gases permanecessem abaixo dos limites estabelecidos pelas normas ambientais.

As emissões de BTEX apresentaram teores em média de $0,00488 \mathrm{mg} \cdot \mathrm{Nm}^{-3}$ que ficou bem abaixo do limite da legislação, uma vez que a legislação $17 . B \operatorname{lmSchV}(24 \mathrm{~h})$ tem o seu limite em14,00 mg. $\mathrm{Nm}^{-3}$. O composto fluoreno foi detectado e quantificado nas emissões de PAHs, cuja concentração foi de $38,5 \mu \mathrm{g} . \mathrm{Nm}^{-3}$ e toxicidade equivalente (TEF) de 0,039 , percentual que ficou abaixo dos limites de emissões da norma Danish EPA.

A Tabela 3 apresenta a análise de metais do char. 
Tabela 3 - Análise de metais do char

\begin{tabular}{cccccc}
\hline mg.kg $\left.^{-1}\right)$ & Char $^{I}$ & NIST2002 & \multicolumn{3}{c}{ Conama 460/2009 } \\
\hline Al & 42300 & n.i. $^{2}$ & Agrícola & Residencial & Industrial \\
\cline { 3 - 5 } Fe & 21800 & 35000 & n.i. & n.i. & n.i. \\
As & 0,076 & 17,7 & 35 & n.i. & n.i. \\
$\mathbf{C u}$ & 100 & n.i. & 200 & 35 & 150 \\
$\mathbf{C r}$ & 100 & 130 & 150 & 400 & 600 \\
$\mathbf{M n}$ & 400 & 538 & 450 & 300 & 400 \\
$\mathbf{Z n}$ & 100 & 106 & n.i. & 1000 & 2000 \\
\hline
\end{tabular}

Char ${ }^{1}$ : Valores representando média dos três ensaios realizados; n..i.': não identificado.

Por meio da Tabela 3, foi observado que os metais (As, $\mathrm{Cu}, \mathrm{Cr}$ e $\mathrm{Mn}$ ) presente no char ficaram abaixo da legislação do Conama 460/2009: As com 0,076 mg. $\mathrm{kg}^{-1}$ com limite agrícola de $35 \mathrm{mg} . \mathrm{kg}^{-1}$; $\mathrm{Cu}$ com $100 \mathrm{mg} \cdot \mathrm{kg}^{-1}$ com limite agrícola de $200 \mathrm{mg} . \mathrm{kg}^{-1}$; Cr com $100 \mathrm{mg} \cdot \mathrm{kg}^{-1}$ com limite agrícola de $150 \mathrm{mg} \cdot \mathrm{kg}^{-1}$ e o Mn com $400 \mathrm{mg} \cdot \mathrm{kg}^{-1}$ com limite agrícola de $450 \mathrm{mg} \cdot \mathrm{kg}^{-1}$. Os teores de Fe e $\mathrm{Zn}$ foram regidos pela legislação americana Nist 2002 (National Institute of Standards and Technology), o Fe com 21800 mg. $\mathrm{kg}^{-1}$ com limite $35000 \mathrm{mg} \cdot \mathrm{kg}^{-1}$ e Zn com $100 \mathrm{mg} \cdot \mathrm{kg}^{-1}$ com limite $106 \mathrm{mg} \cdot \mathrm{kg}^{-1} . \mathrm{O}$ zinco foi o composto que ficou mais próximo ao limite da legislação, porém não excedendo seu limite. $\mathrm{O}$ percentual de Al e As pode agregar valor comercial ao char, que pode ser utilizado como aditivo em materiais cerâmicos, o que seria vantajoso já que os produtos sólidos gerados sempre ficam destinados aos aterros sanitários. O grande problema deste char seria o seu teor de cloro (Tabela 1), que precisaria de um estudo específico, antes de ser utilizado ou até mesmo depositado aos aterros sanitários.

\section{CONCLUSÃO}

Os resíduos caracterizados apresentaram poder calorífico superior entre $17,70-35,44 \mathrm{MJ}_{\mathrm{kg}} \mathrm{kg}^{-1}$ o que designa a possibilidade de queima energética.

A análise elementar mostrou baixos percentuais de enxofre e nitrogênio na faixa de 0,01-0,1\% e 0,1-0,3, respectivamente. Desta maneira, prevê-se menor emissão de $\mathrm{SO}_{2}$ e $\mathrm{NO}_{\mathrm{x}}$ na combustão de $\mathrm{RSU}+\mathrm{CM}$.

A análise imediata potencializa o uso de resíduos como fonte energética. $\mathrm{O}$ teor de material volátil foi alto para todos os resíduos ficando entre $73,75-82,17 \%$, este percentual sugere que a combustão se dá de forma muita rápida.

As análises dos gases de combustão apresentaram teores de $36,47 \mathrm{mg} . \mathrm{Nm}^{-3} \mathrm{CO}, 106,86 \mathrm{mg} . \mathrm{Nm}^{-}$ ${ }^{3} \mathrm{NOx}$ e $18,55 \mathrm{mg} \cdot \mathrm{Nm}^{-3} \mathrm{SO}_{2}$, notando que estes gases geraram percentuais bem abaixo da legislação mais rigorosa: a alemã: 17. BlmSchV $(24 \mathrm{~h})$, que restringem os limites de $70 \mathrm{mg} . \mathrm{Nm}^{-3} \mathrm{CO}, 280$ mg. $\mathrm{Nm}^{-3} \mathrm{NO}_{\mathrm{x}}$ e $70 \mathrm{mg} . \mathrm{Nm}^{-3} \mathrm{SO}_{2}$. As emissões de BTEX e HPA apresentaram percentuais de $0,004875 \mathrm{mg} \cdot \mathrm{Nm}^{-3}$ e $0,039 \mu \mathrm{g} \cdot \mathrm{Nm}^{-3}$, respectivamente, ficando bem abaixo do limite da legislação. 


\section{9 a 22 de outubro de 2014 \\ Florianópolis/SC}

O produto sólido (char) formado apresentou alto percentual de cloro de 4,93\%, uma vez que o processo de pirólise a temperatura de $300{ }^{\circ} \mathrm{C}$ favoreceu a não liberação do cloro do RSU. Desta maneira, pequeno percentual de cloro $0,07 \%$ do cloro original foi liberado na pirólise, diminuindo os riscos de formação de poluente relacionados, como $\mathrm{HCl}$ e dioxinas e furanos.

Logo, a utilização do processo termoquímico: pirólise acoplado ao processo de pós-combustão pode ser bem vantajosa, uma vez que a avaliação preliminar da queima do RSU+CM mostrou que os gases de combustão não excederam o limite da legislação. Uma etapa final deverá ser realizada, no intuito de realizar medição de outros poluentes, tais como dioxinas e furanos e $\mathrm{HCl}$, e realizar um estudo a cima do char formado.

Agradecimentos: A pesquisa foi realizada com parceria da UFSC com a empresa Albrecht Equipamentos Industriais S.A. e com a parceria do Conselho Nacional de Desenvolvimento Científico e Tecnológico-CNPq.

\section{REFERÊNCIAS}

17.BlmSchV - Siebzehnte Verordnung zur Durchführung des Bundes: Immissionsschutzgesetzes. Verordnung über die Verbrennung und die Mitverbrennung von Abfällen (17 Regulamentação para o Cumprimento da Lei Federal Alemã de Controle das Emissões: Regulamentação sobre a combustão e co-combustão de resíduos e materiais combustíveis semelhantes), 2003.

ASTM D2015 - American Society for Testing and Material: Standard Test Methods for Gross Calorific Value of Coal and Coke by the Adiabatic Bomb Calorimet. 2000.

ASTM D4239 - American Society for Testing and Material: Standard Test Methods for Instrumental Determination of Sulphur in Laboratory Samples of Coal, 2002.

ASTM D5373-02 - American Society for Testing and Material: Standard Test Methods for Instrumental Determination of Carbon, Hydrogen and Nitrogen in Laboratory Samples of Coal, 2002.

ASTM E 1131-03 - American Society for Testing and Material: Standard Test Methods for Compositional Analysis by Thermogravimetry. 2004.

CONAMA. Resolução CONAMA n ${ }^{\circ} 316$ de 2002. Gestão de resíduos e produtos perigosos, 2002.

CONAMA. Resolução CONAMA n ${ }^{\circ} 420$ de 2009. Dispõe sobre critérios e valores orientadores de qualidade do solo quanto à presença de substâncias químicas e estabelece diretrizes para o gerenciamento ambiental de áreas contaminadas por essas substâncias em decorrência de atividades antrôpicas. 2009, p. 81-84.

COSTA, M.; DELL'ISOLA, M.; MASSAROTTI, N. Temperature and residence time of the combustion products in a waste-to-energy plant. Fuel, v. 102, p. 92-105, 2012.

Danish Environment Protection Agency. Guidelines for Emission Regulation - Limitation for air pollution. Guideline n.1, 2002. 
DIN EN 15289 - Solid biofuels - Determination of total content of sulfur and chlorine, 2011.

MALKOW, T. Novel and innovative pyrolysis and gasification technologies for energy efficient and environmentally sound MSW disposal. Waste Management, v. 24, p. 56-79, 2004.

MCKAY, G. Dioxin characterisation, formation and minimisation during municipal solid waste (MSW) incineration: review. Chemical Engineering Journal, v. 86, p. 343-368, 2002.

MISKOLCZI, N.; BORSODI, N.; BUYONG, F.; ANGYAL, A.; WILlIAMS, P.T. Production of pyrolytic oils by catalytic pyrolysis of Malaysian refuse-derived fuels in continuously stirred batch reactor. Fuel Processing Technology. v. 92, p. 925-932, 2011.

NIST-Nacional Institute of Standards and Technology. Standard Reference Materials-SRM2709, 2002.

USEPA Compendium Méthod TO - 17 - Determination of Volatile Organic Compounds in Ambient Air Using Active Sampling Onto Sorbent Tube. Environmetal Protection Agency, Washington, D. C.,44.

USEPA Método 4 - Determination of moisture content in stack gases. Environmetal Protection Agency, Washington, D. C.,44.

USEPA Método 23 - Determination of Polichlorinated Dibenzo-p-dioxins and Polychlorinated Dibenzofurans from Municipal waste Combustors. Environmetal Protection Agency, Washington, D. C.,44. 